\title{
Asymptotic behavior of Nonlinear Euler Equation and Its Application
}

\author{
Jifeng Bao ${ }^{1,2, a}$, Xiangqing Zhao ${ }^{1,2, b}$, Yuedan $\operatorname{Jin}^{1, c}$ \\ ${ }^{1}$ Department of Mathematics, Zhejiang Ocean University, Zhoushan, Zhejiang 316022 \\ ${ }^{2}$ Key Laboratory of Oceanographic Big Data Mining \& Application of Zhejiang Province, Zhoushan, \\ Zhejiang 316022

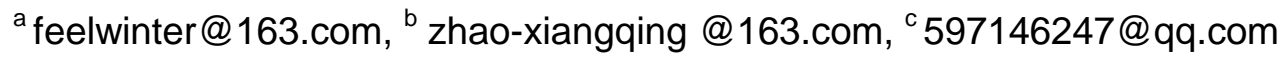

Keywords: Euler equation; Asymptotic behavior; Equilibrium; Ecological models

Abstract: The asymptotic behavior of a class of special nonlinear differential equation with variable coefficient--Euler equation was studied in this paper. We establish the criterion to determine stability of the equilibrium for Euler equation first. Then, we apply the theoretic results to some ecological models to analyze evolution of the certain ecological system.

\section{Introduction}

Euler equation

$$
t^{n} \frac{d^{n} x}{d t^{n}}+a_{1} t^{n-1} \frac{d^{n-1} x}{d t^{n-1}}+\cdots+a_{n-1} t \frac{d x}{d t}+a_{n} x=0
$$

is one of the important higher-order ordinary differential equations which is extensively applied to describe the evolution of ecological system, electronic circulation system, and other physical fields, etc. Although Eq. 1 is high-order ordinary differential equation with variable coefficients which is much complicated in finding analytic solution, its solution formula is established by the transformation $s=\ln t$ which change Eq. 1 into high-order ordinary differential equation with constant coefficients [1]:

$$
\frac{d^{n} x}{d s^{n}}+b_{1} \frac{d^{n-1} x}{d s^{n-1}}+\cdots+b_{n-1} \frac{d x}{d s}+b_{n} x=0
$$

In this paper, we are going to study the asymptotic behavior of equilibrium for Euler equation. Euler equation Eq. 1 can also be changed into the equivalent "Euler equations":

$$
t \cdot \mathbf{X}^{\prime}(t)=\mathbf{A X}(t)
$$

where $\mathbf{X}(t)=\left(x_{1}(t), x_{2}(t), \cdots, x_{n}(t)\right)$ is vector function, $\mathbf{A}=\left(a_{i j}\right)_{n \times n}$ is constant matrix [2]. Intuitively, the asymptotic behavior of equilibrium will sure depend more or less on characteristic roots. In fact, high-order constant coefficient differential equation Eq. 2has the solution of the exponential function $x=e^{\lambda t}$, while Euler equation Eq. 1 has the solution of power function $x=t^{\lambda}$. Based on this observation, Eq. 1 and Eq. 2 have the same asymptotic behavior when $t \rightarrow \infty$ from the point of view of topology. So, the criterion to determine stability of the equilibrium for Euler equation Eq. 1 should be similar to Eq. 2 which determined by characteristic roots. 


\section{Asymptotic behavior analysis}

\section{Linear Euler system}

Lemma 1 Let $\lambda_{1}, \lambda_{2}, \cdots, \lambda_{r}$ be $r$ distinct characteristic roots of matrix $\mathbf{A}$ with multiple numbers $m_{1}, m_{2}, \cdots, m_{r}$ satisfying $m_{1}+m_{2}+\cdots+m_{r}=n$. Let $\mathbf{V}_{i}^{j}\left(j=1,2, \cdots, m_{i}\right)$ are $m_{i}$ linearly independent solutions of

$$
\left(\mathbf{A}-\lambda_{i} \mathbf{E}\right)^{m_{i}} \mathbf{V}_{i}^{j}=0
$$

then the general solution of Eq. 3 is

$$
\mathbf{X}(t)=\sum_{i=1}^{r} \sum_{j=1}^{m_{i}} c_{i j} t^{\lambda_{i}}\left(\sum_{k=0}^{m_{i}-1} \frac{\left(\mathbf{A}-\lambda_{i} \mathbf{E}\right)^{k}(\ln t)^{k}}{k !}\right) \mathbf{V}_{i}^{j},
$$

where

$$
\mathbf{P}_{i j}(t)=\left(\sum_{k=0}^{m_{i}-1} \frac{\left(\mathbf{A}-\lambda_{i} \mathbf{E}\right)^{k} t^{k}}{k !}\right) \mathbf{V}_{i}^{j} .
$$

Proof. On one hand, $\mathbf{X}^{\prime}(s)=\mathbf{A X}(s)$ have solution ${ }^{[1]}$

$$
\mathbf{X}(s)=\sum_{i=1}^{r} \sum_{j=1}^{m_{i}} c_{i j} e^{\lambda_{i} t} \mathbf{P}_{i j}(s)
$$

On the other hand, by the variable transformation $s=\ln t$, i.e. $t=e^{s}$, we have

$$
\frac{d \mathbf{X}}{d t}=\frac{d \mathbf{X}}{d s} \cdot \frac{d s}{d t}=\frac{1}{t} \cdot \frac{d \mathbf{X}}{d s} \Rightarrow t \frac{d \mathbf{X}}{d t}=\frac{d \mathbf{X}}{d s} .
$$

Thus Eq. 3 can be convert to $\mathbf{X}^{\prime}(s)=\mathbf{A X}(s)$.

\section{Theorem 1}

(a) Suppose the real part of all the characteristic roots of matrix $\mathbf{A}$ is negative, then the Null solution of Eq. 3 is asymptotically stable;

(b) Suppose at least one of the real part of characteristic root of matrix $\mathbf{A}$ is positive, then the Null solution of Eq. 3 is unstable.

(c) Suppose the real part of all the characteristic roots of matrix $\mathbf{A}$ is non-positive, but have some zero real part characteristic roots, then the stability for the Null solution of Eq. 3 is relevant to the imaginary part of the characteristic root which has zero real part, but must not be asymptotically stable.

Proof. The general solution Eq. 6 can be rewritten in the following form $\mathbf{X}=\boldsymbol{\Phi}(t) \mathbf{C}$, with

$$
\boldsymbol{\Phi}(t)=\sum_{i=1}^{r} \sum_{j=1}^{m_{i}} t^{\lambda_{i}}\left(\sum_{k=0}^{m_{i}-1} \frac{\left(\mathbf{A}-\lambda_{i} \mathbf{E}\right)^{k}(\ln t)^{k}}{k !}\right) \mathbf{V}_{i}^{j}, \quad \mathbf{C}=\boldsymbol{\Phi}^{-1}\left(t_{0}\right) \mathbf{X}_{0} \quad\left(\text { where } \mathbf{X}\left(t_{0}\right)=\mathbf{X}_{0}\right) .
$$

Proofs of (a)-(c) are just modification of those for higher-order ordinary differential equation Eq. 2 ${ }^{[1,2]}$, so we will prove (a) only in detail.

Suppose the real part of all the characteristic roots of matrix $\mathbf{A}$ is negative, then there 
exists $\sigma>0$ such that $\operatorname{Re} \lambda_{i}<-\sigma,(i=1,2, \cdots, n)$. Thus, there exists a positive number $M>0$, such that for all $t \in\left[t_{0},+\infty\right)$, we have $\left\|\mathbf{P}_{\mathrm{ij}}(t) t^{\lambda_{i} t}\right\|<M \cdot t^{-\frac{\sigma}{2}}$. Thus,

$$
\|\mathbf{X}(t)\| \leq \sum_{i=1}^{r} \sum_{j=1}^{m_{i}}\left|c_{i j}\right| M \cdot t^{-\frac{\sigma}{2}}=\|\mathbf{C}\| M \cdot t^{-\frac{\sigma}{2}} .
$$

Obviously, we have

$$
\|\mathbf{C}\| \leq\left\|\boldsymbol{\Phi}^{-1}\left(t_{0}\right)\right\| \cdot\left\|\mathbf{X}_{0}\right\|
$$

Then, combining Eq. 10 and Eq. 11, we have

$$
\|\mathbf{X}(t)\| \leq\left\|\mathbf{\Phi}^{-1}\left(t_{0}\right)\right\| \cdot\left\|\mathbf{X}_{0}\right\| M \cdot t^{-\frac{\sigma}{2}}
$$

For any fixed $\varepsilon>0$, when taking $\delta=\delta\left(\varepsilon, t_{0}\right)=\left(\varepsilon /\left\|\mathbf{\Phi}^{-1}\left(t_{0}\right)\right\| M\right) t_{0}{ }^{\frac{\sigma}{2}}$, for any $\left\|\mathbf{X}_{0}\right\|<\delta$, we have

$$
\|\mathbf{X}(t)\|<\varepsilon\left(\frac{t_{0}}{t}\right)^{\sigma / 2}
$$

Thus, when $\left\|\mathbf{X}_{0}\right\|<\delta$, for all $t \in\left[t_{0},+\infty\right)$, we have $\|\mathbf{X}(t)\|<\varepsilon$. This means the Null solution $\mathbf{X}(t)=\mathbf{0}$ is stable.

For $\delta=\delta\left(\varepsilon, t_{0}\right)=t_{0}^{\sigma / 2} /\left\|\mathbf{\Phi}^{-1}\left(t_{0}\right)\right\| M$, if $\left\|\mathbf{X}_{0}\right\|<\delta$, then $\|\mathbf{X}(t)\|<\left(t_{0} / t\right)^{\sigma / 2}$. This implies

$\lim _{t \rightarrow \infty}\|\mathbf{X}(t)\|=0$

Thus, the Null solution $\mathbf{X}(t)=\mathbf{0}$ is asymptotically stable.

\section{Nonlinear Euler system}

For nonlinear system

$t \mathbf{X}^{\prime}=f(\mathbf{X})$.

with $f(\mathbf{0})=\mathbf{0}$. By Talyor's formula, we get $f(\mathbf{X})=\mathbf{A} \mathbf{X}+R(\mathbf{X})$, with $\lim _{\mid \mathbf{X} \| \rightarrow 0} R(\mathbf{X}) /\|\mathbf{X}\|=0$.

Then the stability for the nonlinear system Eq. 15 can be characterized by its first-order approximate linear system $t \mathbf{X}^{\prime}=A \mathbf{X}$. In fact, we have the following theorem.

\section{Theorem 2}

(a) Suppose the real part of characteristic roots of matrix $\mathbf{A}$ are all negative, then the Null solution of Eq. 15 is asymptotically stable.

(b) Suppose the real part of at least one characteristic root of matrix $\mathbf{A}$ is positive, then the Null solution of Eq. 15 is not stable.

Proof. Similar that of higher-order ordinary differential equation, we'll omit the detail.

\section{Applications in ecological system}

With the high speed development of economic, pollution gradually hurts the ecological system day by day. We cannot take a blind eye to the worsen of our survival environment. The evolution of 
ecological system is always described by ordinary differential systems ${ }^{[1,2]}$. As claimed in [4], some certain ecological model can be described by Euler equations. After being modeled by ordinary differential equations, the final evolution trend of a certain ecological system is exactly the asymptotic behavior of the equilibrium of the differential equation, thus asymptotic behavior is very important for ecological system.

Example 1. Study the stability of the following ecological system ${ }^{[4]}$ :

$$
\left\{\begin{array}{l}
\frac{d x}{d t}=-2 x+y+x e^{x}, \\
\frac{d y}{d t}=x-y+x^{3} y .
\end{array}\right.
$$

$$
\left\{\begin{array}{l}
t \frac{d x}{d t}=-2 x+y+x e^{x} \\
t \frac{d y}{d t}=x-y+x^{3} y
\end{array}\right.
$$

Solution. The first-order approximate systems are

$$
\left\{\begin{array} { l l } 
{ \frac { d x } { d t } = - 2 x + y , } \\
{ \frac { d y } { d t } = x - y , }
\end{array} \quad \left\{\begin{array}{l}
t \frac{d x}{d t}=-2 x+y \\
t \frac{d y}{d t}=x-y
\end{array}\right.\right.
$$

The characteristic roots are $\lambda_{1,2}=-3 \pm \sqrt{5} / 2$, thus Eq. 16 and Eq. 17 are asymptotically stable.

Remark 1. We have mentioned in the introduction about the evolution trend of Eq. 1 and Eq. 2, we illustrate it numerically here for the sake of clearness.

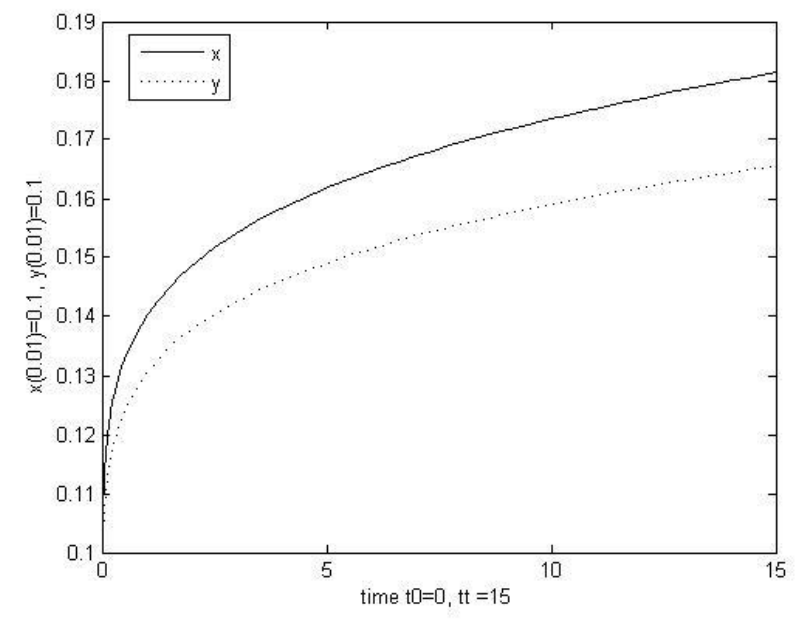

Fig. 1 Integral curve of Eq. 17

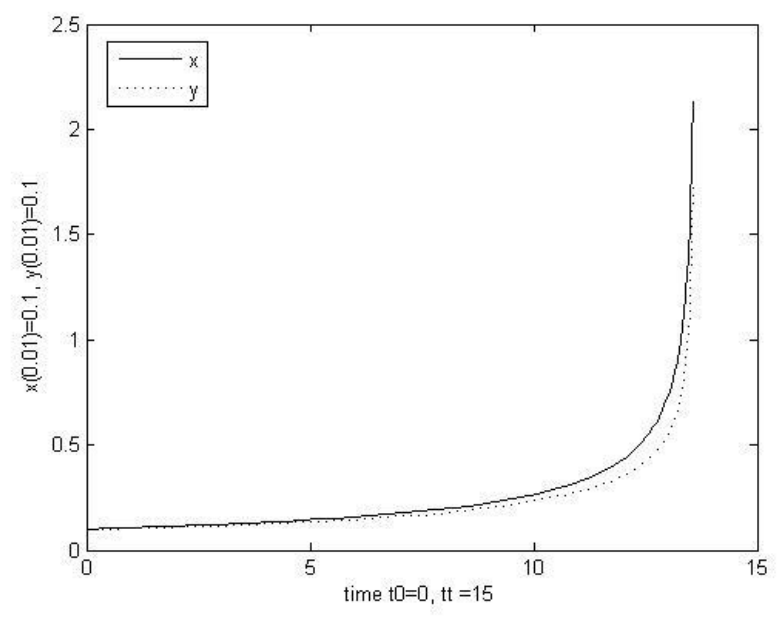

Fig. 2 Integral curve of Eq. 18

Example 2. Study the stability of the Null solution of the ecological system ${ }^{[4]}$ :

$$
3 t^{2} \frac{d^{2} x}{d t^{2}}+t \frac{d x}{d t}+x=0
$$

Solve: First, we convert this Euler equation to the first-order Euler system:

$$
\left\{\begin{array}{l}
t \frac{d x_{1}}{d t}=x_{2} \\
t \frac{d x_{2}}{d t}=-\frac{1}{3} x_{1}+\frac{2}{3} x_{2}
\end{array}\right.
$$


The characteristic roots are $\lambda_{1}=1 / 3, \lambda_{2}=-1$, thus, the Null solution is not stable.

\section{Acknowledgements}

This work was supported by the National Natural Science Foundation of China (grants 11505154) and Natural Science Foundation of Zhejiang province (grants LQ16A010003).

\section{Referrences}

[1] G.X. Wang, Z.M. Zhou, S.M. Zhu, Ordinary Differential Equations (Third Edition) [M], Beijing: Higher Education Press (2007).

[2] S.L. Cai, Ordinary Differential Equations (Second Edition) [M], Wuhan: Wuhan University Press (2007).

[3] X.Q. Xie, X.Q. Zhao, A.M. Liu, Solution of Inhomogeneous Euler Equations, Studies in College Mathematics, accepted.

[4] C.J. Sun, Binomial Coefficient based Euler Equation ecological population model and its degeneration. Journal of Biomathematics, 28 (3), pp. 493-498 (2013). 